\title{
Net effects of multiple stressors in freshwater ecosystems: a meta-analysis
}

\author{
Jackson, M.C. ${ }^{1 * \dagger}$, Loewen, C.J.G. ${ }^{2 \dagger}$, Vinebrooke, R.D. ${ }^{2}$, Chimimba, C.T. ${ }^{1}$ \\ ${ }^{1}$ Centre for Invasion Biology, Department of Zoology and Entomology, University of Pretoria, P/Bag X20, \\ Hatfield, 0028 South Africa \\ ${ }^{2}$ Department of Biological Sciences, University of Alberta, Edmonton, Alberta, T6G 2E9 Canada
}

\footnotetext{
* Corresponding author: mjackson@zoology.up.ac.za; +27(0)715171898

${ }^{\dagger}$ These authors contributed equally to this work
}

\section{Type of paper: Review}

\begin{abstract}
The accelerating rate of global change has focused attention on the cumulative impacts of novel and extreme environmental changes (i.e., stressors), especially in marine ecosystems. As integrators of local catchment and regional processes, freshwater ecosystems are also ranked highly sensitive to the net effects of multiple stressors, yet there has not been a large-scale quantitative synthesis. We analysed data from 88 papers including 286 responses of freshwater ecosystems to paired stressors, and discovered that overall, their cumulative mean effect size was less than the sum of their single effects (i.e., an antagonistic interaction). Net effects of dual stressors on diversity and functional performance response metrics were additive and antagonistic, respectively. Across individual studies, a simple votecounting method revealed that the net effects of stressor pairs were frequently more antagonistic (41\%) than synergistic (28\%), additive $(16 \%)$ or reversed $(15 \%)$. Here, we define a reversal as occurring when the net impact of two stressors is in the opposite direction (negative or positive) from that of the sum of their single effects. While warming paired with nutrification resulted in additive net effects, the overall mean net effect of warming combined
\end{abstract}


with a second stressor was antagonistic. Most importantly, the mean net effects across all stressor pairs and response metrics were consistently antagonistic or additive, contrasting the greater prevalence of reported synergies in marine systems. Here, a possible explanation for more antagonistic responses by freshwater biota to stressors is that the inherent greater environmental variability of smaller aquatic ecosystems fosters greater potential for acclimation and co-adaptation to multiple stressors.

Keywords: antagonism, biodiversity, climate change, cumulative impacts, ecological surprises, functional resistance, reversals, synergy.

\section{Introduction}

The rise of "ecological surprises" in the primary scientific literature highlights the growing uncertainty over the cumulative impacts of multiple novel and extreme environmental changes, or "stressors" (e.g., Paine et al., 1998; Christensen et al., 2006; Lindenmayer et al., 2010; Dehedin et al., 2013; Harvey et al., 2013). There is increasing evidence from marine environments that these stressors, such as rising temperatures, biological invasions and habitat destruction, act synergistically to exacerbate biodiversity loss and ecological degradation (Crain et al., 2008; Harvey et al., 2013; Przeslawski et al., 2015). Interactions among stressors are at the core of these unexpected net ecological impacts (Sala et al., 2000) as they can generate complex effects that lessen or amplify the direct single effect of each stressor. The reported prevalence of non-additive effects of stressors across many marine ecosystems (Crain et al., 2008; Darling \& Cote, 2008; Harvey et al., 2013; Ban et al., 2014) attests to an urgent need to fill knowledge gaps in freshwater ecosystems (Root et al., 2003; Ormerod et al., 2010; Staudt et al., 2013; Hering et al. 2015).

Empirical evidence of the net effects of multiple stressors on freshwaters remains very 
limited (but see Christensen et al., 2006; Darling \& Cote, 2008; Mantyka-Pringle et al., 2014) despite their impacts being greatest on freshwater biodiversity (Jenkins, 2003; WWF, 2014). Freshwater ecosystems are particularly vulnerable to global change (Dudgeon et al., 2006; Ormerod et al., 2010) as they often occupy low points in landscapes, integrating the effects of local catchment and regional atmospheric processes (Williamson et al., 2009). In comparison, recent meta-analyses of the marine literature show that the net impact of multiple stressors are frequently either greater than (i.e., a synergistic interaction; Crain et al., 2008; Harvey et al., 2013) or equal to (i.e. an additive effect; Ban et al., 2014; Strain et al., 2014) the sum of their single effects. Net effects of two or more stressors that were less than the potential additive outcome (i.e., an antagonistic interaction) were less common (Crain et al., 2008; Harvey et al., 2013). Such variation in the net effects of stressor combinations depends in part on how impact is measured, as different biological receptors will inherently vary in their responsiveness to environmental change (termed response diversity; Elmqvist et al., 2003). For example, compensatory species dynamics within a stressed community may result in measurable changes in biodiversity while muting changes in function (e.g., primary production; Vinebrooke et al., 2003).

Theoretical models that predict the combined impact of stressor pairs on populations or communities are often based on an evaluation of the similarity of their independent impacts (Vinebrooke et al., 2004). For instance, if stressors A and B are highly redundant and both extirpate or negatively influence the same set of species in a community, then their net impact on species richness or functional performance (e.g., productivity or abundance) should be less than the sum of their independent effects (an antagonistic interaction). In contrast, synergy between stressors A and B can occur if species are affected only upon exposure to both stressors, resulting in their combined impact being greater than the sum of their single effects (a synergistic interaction). If stressor A affects a different set of species than stressor 
$B$, then their net impact on the community can equal the sum of their direct effects (an additive effect). In some cases, the net effect of stressors A and B may actually be in the opposite direction (positive or negative) than predicted based on their independent effects (Piggot et al., 2015). For instance, Christensen et al. (2006) found that warming reversed the positive effect of acidification on phytoplankton. We term such interactions as 'reversals', perhaps representing the greatest of all 'ecological surprises'.

Here, we synthesise findings from dual-stressor studies in freshwater ecosystems to address two main questions: (1) what is the cumulative mean interaction and frequency of interaction types across all studies?; and (2) how do interactions vary among response metrics and stressor pairs? We also focused on how higher temperatures associated with climate change interact with other key stressors to impact ecosystem properties. We used a metaanalytical approach to optimise our ability to both conduct a powerful quantitative test of the nature of interactions between stressors affecting freshwater ecosystems and identify testable hypotheses (Gurevitch et al., 2000; Parmesan et al., 2013; Hillebrand \& Gurevitch, 2014).

\section{Materials and methods}

\section{Data selection}

We searched the primary scientific literature and identified papers in which the impacts of multiple stressors were compared, both in combination and alone, to a non-stressed control (see Supporting Information 1 for full search terms and methods). Reported stressors included acidification, higher temperatures, ultraviolet radiation (UVR), contamination (xenobiotics or salinity), nutrification, habitat alteration (physical manipulation, sedimentation, altered flow regime or drought) and invasive species. We considered the following response currencies or metrics: (i) survival, (ii) growth/size, (iii) condition, (iv) reproductivity, (v) behaviour, (vi) total biomass/abundance, (vii) diversity, and (viii) leaf 
decomposition.

We used the term 'observation' to refer to individual responses used in our analyses, and the term 'paper' to refer to their source documents. In several cases, multiple observations were extracted from individual papers when either several experiments were conducted (i.e., using different sets of species, study locations or stressor combinations) or various organismal groups were measured (e.g., producers, invertebrates or vertebrates). If the response of a specific organismal group to dual stressors during a single experiment was assessed using multiple metrics (e.g., plant biomass and plant diversity), then we treated each as an independent observation for inclusion only in our 'full dataset' $(n=286)$. The full dataset was then used for our mixed effects response metric meta-analyses (detailed and pooled; Table 1). For the remainder of our comparisons, we excluded all diversity metrics ( $n$ $=31$ ) and reduced our dataset to include only the most inclusive response metrics per experiment for each organismal group. For experiments where multiple response metrics were reported, the most inclusive response metric was selected where community responses were preferred over population or organism-level responses, and metrics were selected in favour of biomass/abundance over survival, survival over growth/size, growth/size over condition, condition over reproductivity and reproductivity over behaviour. However, if the same experiment measured impact separately on multiple organism groups (e.g., producers and invertebrates), then each observation was retained. This 'most inclusive response metric dataset' $(n=230)$ was used for the majority of our meta-analyses (i.e., those not specifically comparing response metrics; Table 1) to minimize data non-independence. See Table S1 (Supporting Information 2) for a complete list of observations included in each dataset. For each observation/stressor response, we extracted mean, standard deviation and sample size values for each treatment combination (stressor A; stressor B; stressor A and B; no stressor control). We also collected relevant categorical data (e.g., location and response metric used 
to measure impact) for each observation (Table S1).

Table 1. Datasets used for each categorical analysis (meta-analytic and vote counting) and the levels of each category (where $\mathrm{n} \geq 8$ ). See Table $\mathrm{S} 2$ in Supporting Information 2 for full model terms.

\begin{tabular}{|c|c|}
\hline Dataset & Categorical analyses \\
\hline \multirow[t]{2}{*}{ Full dataset $(n=286)$} & Detailed response metric: \\
\hline & $\begin{array}{l}\text { Animal survival; Animal growth/size; Plant growth/size; Animal } \\
\text { condition; Animal biomass/abundance; Plant biomass/abundance; } \\
\text { Animal diversity; Plant diversity; Leaf decomposition }\end{array}$ \\
\hline \multirow[t]{2}{*}{ Full dataset $(n=286)$} & $\underline{\text { Pooled response metric: }}$ \\
\hline & Diversity; Functional Performance \\
\hline \multirow{2}{*}{$\begin{array}{l}\text { Most inclusive response metric } \\
\text { dataset }(n=230)\end{array}$} & Level of biological organisation: \\
\hline & Community; Population; Organism \\
\hline \multirow{2}{*}{$\begin{array}{l}\text { Most inclusive response metric } \\
\text { dataset }(n=230)\end{array}$} & Organism group: \\
\hline & Vertebrate; Invertebrate; Producer \\
\hline \multirow{2}{*}{$\begin{array}{l}\text { Most inclusive response metric } \\
\text { dataset }(n=230)\end{array}$} & $\underline{\text { Stressor pair: }}$ \\
\hline & $\begin{array}{l}\text { Contamination x Habitat Alteration; Contamination x Invasion; } \\
\text { Contamination x Nutrification; Contamination x Warming; Habitat } \\
\text { Alteration x Nutrification; Invasion x Invasion; Invasion x } \\
\text { Nutrification; Nutrification x UVR; Nutrification x Warming; } \\
\text { Warming x UVR }\end{array}$ \\
\hline
\end{tabular}

\section{Effect size calculations}

Interaction effect sizes were calculated for each observation in our dataset using Hedges $d$, an estimate of the standardised mean difference not biased by small sample sizes (Gurevitch \& Hedges 2001). The interaction effect size for each observation was calculated by comparing the null predicted additive effect to the actual observed effect of both stressors. Each interaction effect size was therefore based on the absolute difference between the observed net impact of dual stressors against a hypothetical additive outcome based on the sum of their single independent effects (see Supporting Information 1 for equation details). 


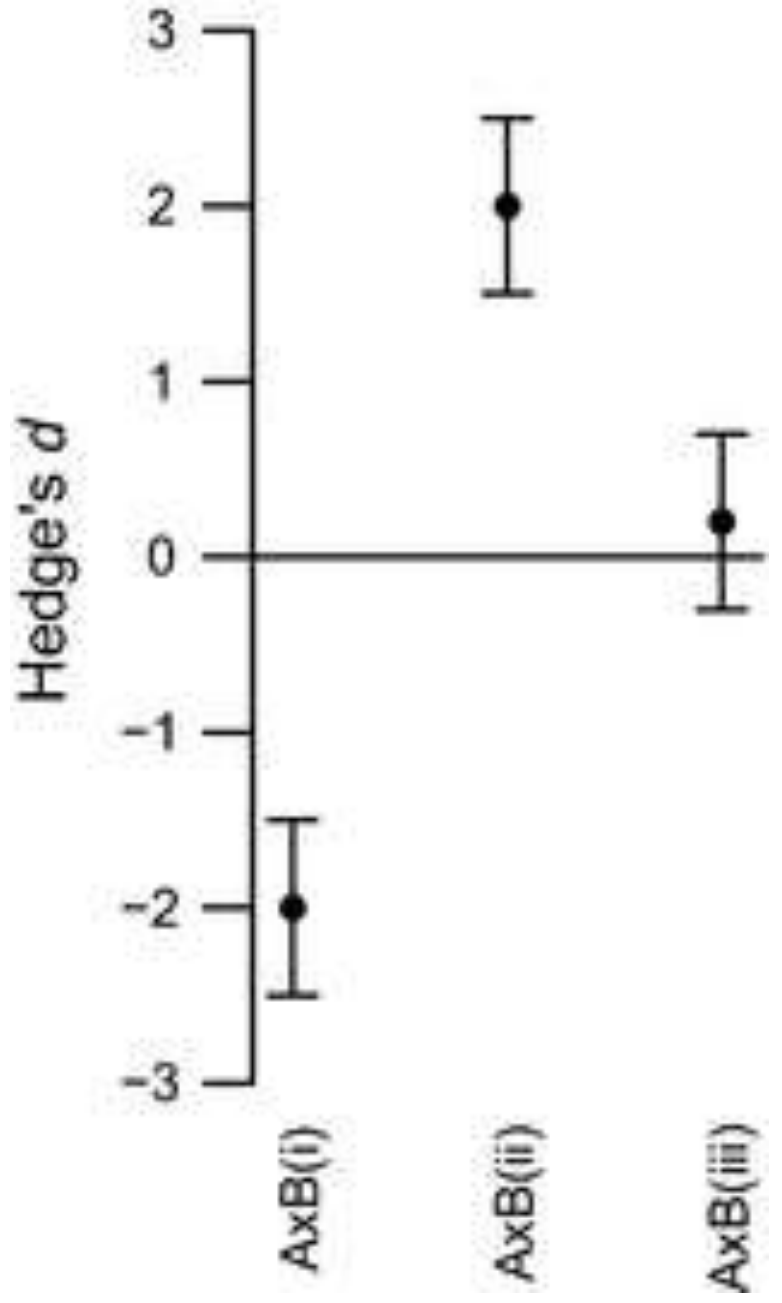

Fig. 1 The theoretical interactive effects of stressors A and B applied in combination, relative to their predicted additive response $(=0)$. Negative effect sizes (less than zero) represent antagonism or reversals (i) and positive effect sizes (greater than zero) represent synergistic interactions (ii), but only if their confidence intervals do not cross the $\mathrm{x}$-axis. Interaction effect sizes with confidence intervals that overlap with zero were considered to be additive (iii).

We inverted the response direction $(-/+)$ of interaction effect sizes for which the additive effects were negative (i.e., where both single effects were negative, or if in opposing directions, where the negative effect had the higher absolute value; Piggot et al., 2015). This allowed us to compare interaction effect sizes regardless of their directionality (Piggot et al., 2015). This means that an effect size $(d)$ of zero represents an exact additive effect of the two stressors (i.e., their combined impact is equal to the sum of their single effects), while a 
positive $d$ denotes a synergistic interaction (a combined impact greater than the sum of their single effects) and a negative $d$ reflects either antagonism or a reversal interaction (a combined impact less than the sum of their single effects; Fig. 1). To distinguish between antagonistic and reversal interactions, we compared the direction (negative or positive, relative to the control) of the observed response to both stressors applied in combination with the direction of their predicted additive response, and assigned reversals where they were opposite. Interaction significance was assessed using 95\% confidence intervals calculated around each effect size, such that any interactions with intervals crossing zero were deemed additive (Fig. 1).

\section{Statistical analyses}

Mean interaction effect sizes across studies were estimated from weighted metaanalyses. In each analysis, 'Observation ID' was treated as a random effect to account for the random component of effect size variation among observations and calculate inverse unconditional variance effect size weights (Gurevitch \& Hedges 2001; see Supporting Information 1 for equations and model details). In addition to using random effects metaanalyses to assess the global mean interaction effect sizes across all observations included in our 'full' and 'most inclusive response metric' datasets, we conducted a series of mixed effects meta-analyses where selected categorical moderators were treated as fixed effects to assess mean interactions at each level of each category (where $n \geq 8$; see Table S2 in Supporting Information 2 for model terms).

Using our 'full dataset', we conducted a detailed response metric analysis to evaluate the sensitivity of different response metrics to multiple stressors (Table 1). We followed this with a pooled response metric analysis, where response metrics were reassigned as either 'diversity' (plant or animal diversity) or 'functional performance' (all other response metrics 
considered), to assess the sensitivities of these broader response categories. We then used our reduced 'most inclusive response metric dataset' to estimate mean effect sizes across receptor categories (response levels and organism groups) and stressor-pair combinations (Table 1). Percentile bootstrapped $95 \%$ confidence intervals were calculated around each mean interaction effect size to assess significance (Fig. 1). Similar to the assessment of interaction effect sizes for single observations, a positive mean effect reflects synergy, a negative mean effect reflects antagonism (reversals could not be distinguished with this method) and cases where the confidences intervals crossed zero were deemed additive.

In addition to the quantitative synthesis described above, we complemented each meta-analytic model with a vote-counting analysis to describe the frequencies of interaction types (including reversals) across individual observations. Randomisation tests of independence (Monte Carlo approximation using 9,999 permutations) were used to assess whether the frequencies of interaction types differed significantly among levels of each categorical moderator where $n \geq 8$ (Table 1$)$.

Weighted meta-analyses were conducted in MetaWin version 2.1 (Rosenberg et al., 2000) and the $\mathrm{R}$ computing program was used to perform independence tests and create figures (R Core Team, 2014). To assess the robustness of our results, we conducted several additional analyses to investigate potential publication bias and the sensitivity of our findings to variation in sample sizes and effect size outliers (Supporting Information 3). Although we found some evidence of asymmetry around our overall mean effect size estimate, we suspect this may be at least partially attributable to the considerable data heterogeneity observed. Nevertheless, the results of our sensitivity analyses indicate that our meta-analytic findings are robust to such variations. 


\section{Stressor interactions across response metrics}

We found 88 articles representing 286 separate observations or biological responses to multiple stressors that met our selection criteria (Table S1). In addition, 11 articles fitting our criteria were not included because we were unable to extract the data or the study did not report margins of error (listed in Supporting Information 2). The majority of the research was carried out in North America (46 of 88 articles), followed by Europe (30) and New Zealand (7). All of the studies were conducted experimentally in laboratories (57), outdoor mesocosms (210) or in situ (19).

Individual observations in our full dataset were most frequently antagonistic (40\%; compared with $26 \%$ synergistic, $19 \%$ additive and $15 \%$ reversed) and the mean interaction effect size across all responses was also significantly less than additive (i.e., antagonistic; Table S2). Multiple stressors exerted significant antagonistic effects on animal abundance/biomass, animal condition, animal growth/size, animal survival and plant diversity (Fig. 2a). Additive mean stressor effects were identified for the other four response metrics (decomposition, animal diversity, plant abundance/biomass and plant growth/size; Fig 2a).

One possible explanation for widespread antagonistic interactions between freshwater stressors involves asymmetry of their single effect sizes. Here, the larger magnitude of the worst stressor completely overrides the effect of the weaker stressor, thereby negating its contribution to their net impact (Folt et al., 1999; Sala et al., 2000). The detected prevalence of antagonisms also suggests that exposure to one stressor often results in greater tolerance to the other (Vinebrooke et al., 2004). Here, a potential mechanism involves hard selection for tolerant organisms that are co-adapted to both stressors, thereby reducing their combined impact. Alternatively, acclimation to each stressor may involve the same behavioural or physiological mechanism, which would result in exposure to one stressor inducing greater tolerance against the other. 

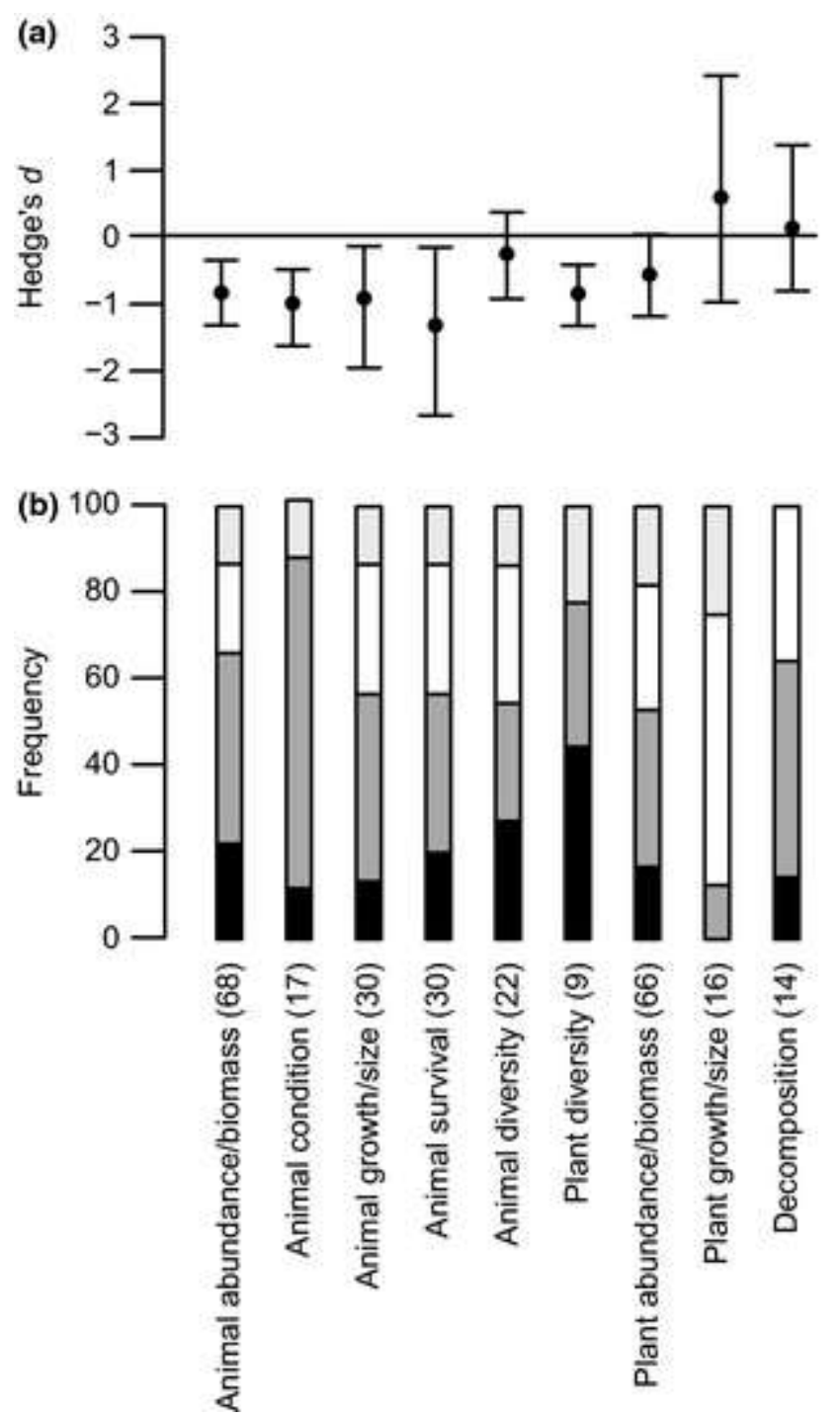

Fig. 2 The mean interaction effect sizes (Hedge's $d$ and bootstrapped 95\% confidence intervals; a) and frequencies (\%) of interaction types (b) for different response metric categories. Interaction types are additive (black), antagonistic (dark grey), synergistic (white) and reversals (light grey). The number of observations/studies included in each category is indicated in parentheses. Mean responses only presented where $\mathrm{n} \geq 8$.

Frequencies of interaction types varied significantly $\left(\chi^{2}=40.36 ; P=0.019 ;\right.$ d.f. $=24$; $n=272$ ) and non-additive interactions were collectively more common than simple additive scenarios. Antagonisms occurred most often with animal condition $(76.47 \%)$, synergies and reversals with plant growth/size (62.50\% and $25.00 \%$, respectively), and additive effects with plant diversity (44.44\%; Fig. 2b). The highly variable nature of stressor interactions across 
these response metrics highlights the importance of currency selection when quantifying the net ecological impact of multiple stressors.

Stressors also exerted differing interactive and additive effects on functional performance and diversity responses, respectively. The mean interaction effect size for functional performance responses was antagonistic, while the mean effect of stressors on diversity was additive. Additive and reversal interactions occurred most frequently with diversity metrics $(32.25 \%$ and $16.13 \%$, respectively) while antagonistic and synergistic interactions occurred more frequently with functional performance metrics $(41.57 \%$ and $27.06 \%$, respectively); however, the frequencies of interaction types did not differ significantly $\left(\chi^{2}=4.87, P=0.174\right.$; d.f. $\left.=3 ; n=286\right)$.

Compensatory species dynamics may explain the different mean interactive effects observed for stressor impacts on freshwater diversity and functional performance. The frequency of additive responses by diversity to dual stressors suggests that species eliminated by one stressor were often not the same that are eliminated by a second stressor. However, the prevalence of antagonism at the functional performance level suggests the remaining tolerant species may often compensate functionally for species loss, thereby reducing the net functional consequences of the stressors. Although the prevalence of functional species compensation has been debated in the literature (Houlahan et al., 2007; Gonzalez \& Loreau, 2009), several lines of evidence show it can help stabilise stressed freshwater communities (e.g., Klug et al., 2000; Fischer et al., 2001; Vinebrooke et al., 2003; Downing et al., 2008). Our findings support how functional resistance to stressors is not simply a function of biodiversity, but more often indicative of species identity and associated traits (e.g., Smith \& Knapp, 2003; Vaz-Pinto et al., 2013). Thus, functional resistance should be related to the response diversity and functional redundancy within stressed communities (Elmqvist et al., 2003; Nyström, 2006; Mori et al., 2012). As a result, our findings point to freshwater 
biodiversity being more sensitive than functioning to the cumulative impacts of multiple stressors.

\section{Stressor interactions across receptor categories}

For analyses of receptor categories and stressor pairs (see following section), we considered only the most inclusive response metrics to avoid pseudo-replication. As a result, our dataset was reduced to 230 observations for these analyses (Table 1; Table S1). The majority of the observations examined responses at the community level and the most frequently examined organisms were invertebrates (Fig. 3). The global mean interaction effect size was significantly antagonistic (Table S2) and of the 230 observations considered, $94(40.87 \%)$ were antagonistic, $64(27.83 \%)$ were synergistic and 34 (14.78\%) were reversals, while $38(16.52 \%)$ were additive.

The cumulative mean interaction effect of stressors was significantly antagonistic at the community and organismal level but additive at the population level (Fig. 3a; Table S2). However, the frequencies of interaction types did not differ significantly among levels of biological organisation $\left(\chi^{2}=11.39 ; P=0.074 ;\right.$ d.f. $\left.=6 ; n=230\right)$. While antagonistic interactions were most frequent at the organismal (65.22\%) and community (40.88\%) levels of biological organisation, synergies and reversals occurred most frequently at the population level (37.14\% and $17.14 \%$, respectively) and additive interactions were most common at the community level (18.98\%; Fig. 3b).

Dual stressors exerted significant antagonistic effects on invertebrates and vertebrates, while primary producers responded in an overall additive fashion (Fig. 3c; Table S2). However, frequencies of interaction types were similar across all organismal groups $\left(\chi^{2}=5.70 ; P=0.457 ;\right.$ d.f. $\left.=6 ; n=224\right)$. Antagonistic responses occurred most frequently for invertebrates (45.21\%) and vertebrates (46.43\%), synergies and reversals were most common 

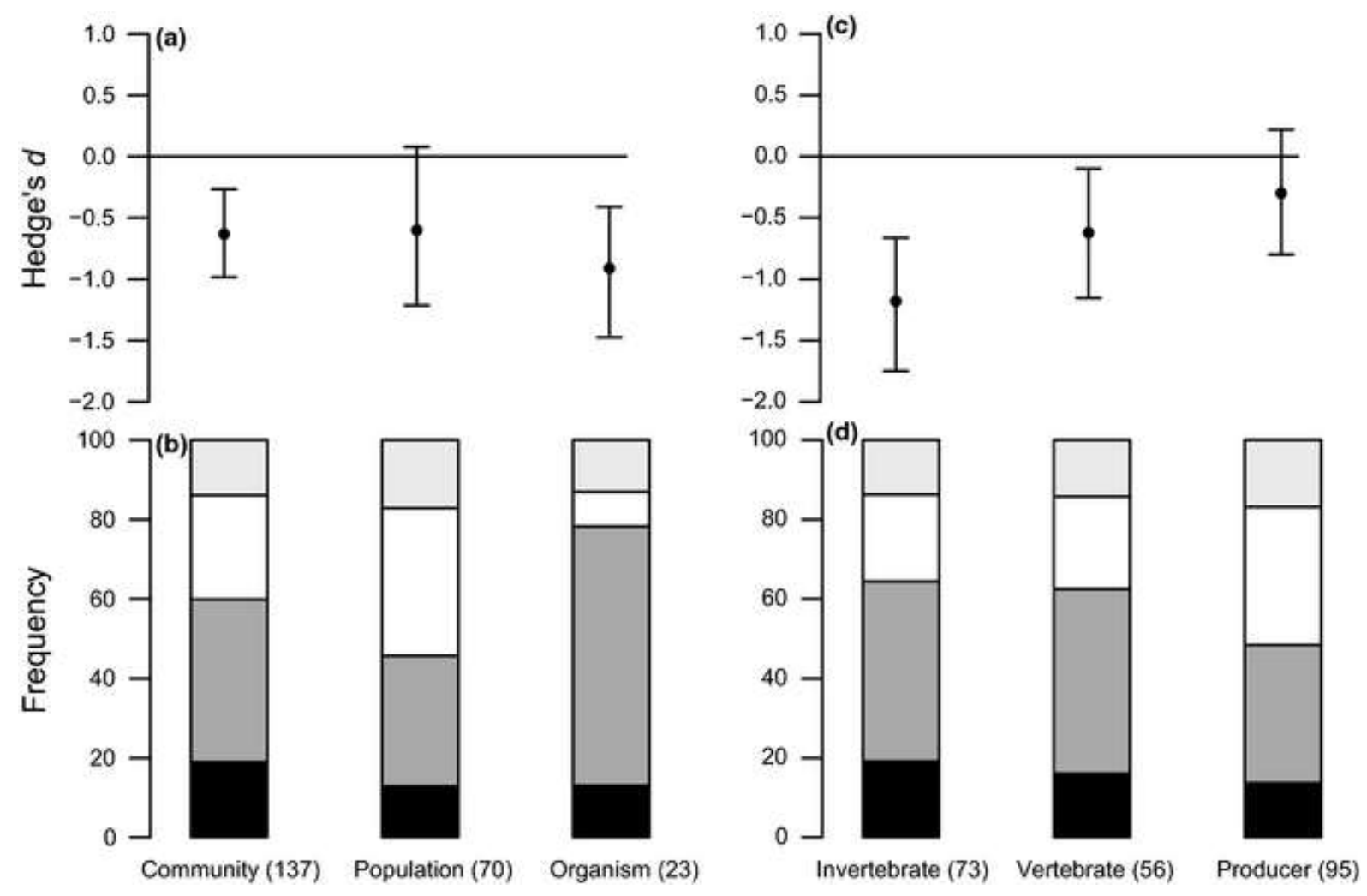

Fig. 3 The mean interaction effect sizes (Hedge's $d$ and bootstrapped 95\% confidence intervals; a, c) and frequencies $(\%)$ of interaction types $(\mathbf{b}, \mathbf{d})$ for different receptor categories, including level of biological organization (a, b) and organism group $(\mathbf{c}, \mathbf{d})$. Interaction types are additive (black), antagonistic (dark grey), synergistic (white) and reversals (light grey). The number of observations/studies included in each category is indicated in parentheses. Mean responses only presented where $\mathrm{n} \geq 8$.

with primary producers $(34.74 \%$ and $16.84 \%$, respectively), and additive interactions most often affected invertebrates (19.18\%; Fig. 3d). These results were surprising because sensitivity to global change is often thought to increase with trophic position (e.g., Crain et al., 2008; Petchey et al., 1999), particularly with warming, as metabolic demands increase faster than ingestion rates with higher temperatures (Vucic-Pestic et al., 2011). Here, the different responses of consumers and primary producers highlight the potential for multiple stressors to weaken trophic interactions, and promote algal blooms. Many of the synergistic responses by primary producers involved net positive effects by stressors such as nutrification, UVR and warming. In fact, 36 of the 64 synergistic interactions in our analysis 
were positive, and of these, 21 showed an increase in producer performance. Globally, correlative evidence suggests that nutrients and climate interact synergistically to increase the overall percentage of cyanobacteria in shallow lakes (Kosten et al., 2012). Experimental evidence supports these observations, showing warming and nutrient enrichment can exert a synergistic positive effect on phytoplankton growth (e.g., Doyle et al., 2005).

\section{Stressor interactions across stressor pairs}

Ten stressor pairs had sufficient observations $(n \geq 8)$ for a comparison of their mean interaction effects (Table 2), which varied with their identity (Fig. 4a). Net effects were significantly antagonistic for contamination $\mathrm{x}$ invasion, contamination $\mathrm{x}$ warming and warming x UVR; however, effects were additive for the remaining seven stressor pairs, including nutrification paired with warming, habitat alteration, invasion and UVR (Fig. 4a).

Table 2 The number of independent observations/studies meeting our criteria used in the stressor pair analysis $(n=230)$.

\begin{tabular}{|c|c|c|c|c|c|c|c|}
\hline & 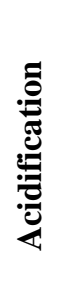 & 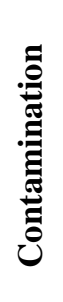 & 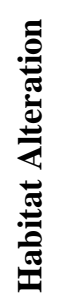 & 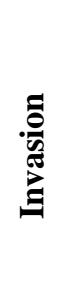 & 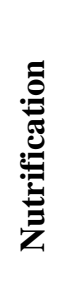 & $\stackrel{\stackrel{2}{3}}{3}$ & 茴 \\
\hline Acidification & 0 & 3 & 2 & 0 & 0 & 3 & 5 \\
\hline Contamination & & 6 & 19 & 11 & 14 & 6 & 33 \\
\hline Habitat Alteration & & & 4 & 2 & 21 & 1 & 6 \\
\hline Invasion & & & & 13 & 10 & 0 & 7 \\
\hline Nutrification & & & & & 0 & 10 & 41 \\
\hline UVR & & & & & & 0 & 13 \\
\hline Warming & & & & & & & 0 \\
\hline
\end{tabular}


pairs $\left(\chi^{2}=28.25 ; P=0.402 ;\right.$ d.f. $\left.=27 ; n=185\right)$, antagonistic effects occurred most frequently Although the frequencies of interaction types were not significantly different among stressor when warming occurred with UVR (61.54\%), synergistic interactions occurred most often with nutrification and UVR $(50.00 \%)$, reversal interactions were linked with warming and nutrification (26.83\%) and additive interactions were common with paired invasions (30.77\%; Fig. 4b).

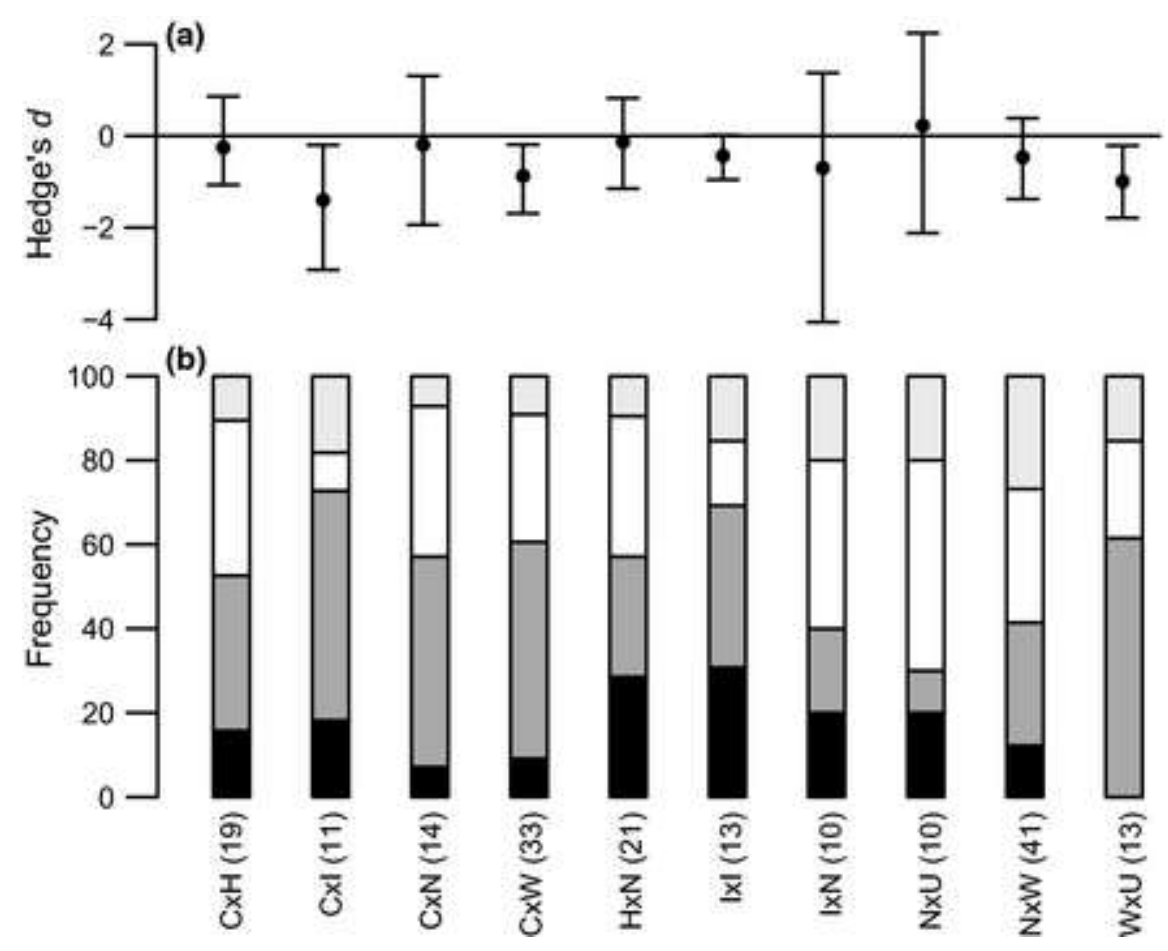

Fig. 4 The mean interaction effect sizes (Hedge's $d$ and bootstrapped 95\% confidence intervals; a) and frequencies $(\%)$ of interaction types (b) for different stressor-pair combinations. Interaction types are additive (black), antagonistic (dark grey), synergistic (white) and reversals (light grey). The number of observations/studies included in each category is indicated in parentheses. Mean responses only presented where $\mathrm{n} \geq 8 . \mathrm{W}=$ warming; $\mathrm{C}=$ contamination; $\mathrm{H}=$ habitat alteration; $\mathrm{I}=$ invasion; $\mathrm{N}=$ nutrification; and $\mathrm{U}=$ ultraviolet light radiation.

When higher temperature interacted with a second freshwater stressor, the mean interaction was antagonistic overall $(d=-0.68 ; 95 \% \mathrm{CI}=-1.1$ to $-0.3 ; n=105)$. This finding 
is in contrast to studies of marine ecosystems where both Crain et al., (2008) and Harvey et al., (2013) found that warming most often interacted with a second stressor to produce a synergistic response. However, a recent re-analysis of the data presented by Crain et al. (2008) suggests that their original methods may have overrepresented synergies (Piggott et al., 2015). Furthermore, Ban et al., (2014) found that the mean effect of multiple stressors in coral reefs was additive overall, and it is important to note that different ecosystem types face different combinations of key stressors (Jenkins, 2003; Pratchett et al., 2011). Lake (1990) suggested that benthic communities in freshwater and marine ecosystems may react differently to certain disturbances because of differences in the proportion of mobile versus sedentary biota. More general differences between freshwater and marine responses may be based on how specific stressors interact with inherent ecosystem properties. For example, Bancroft et al., (2007) predicted that UVR impacts should vary between marine and freshwater environments owing to differing optical qualities of the water; however, they were unable to detect significant differences from their meta-analysis. Additionally, the effects of some stressors (e.g., salinity and metal contaminants) may differ among freshwater and marine receptors based on physiological differences between biota (Hall \& Anderson, 1995; Heugens et al., 2001).

Higher environmental variability of smaller aquatic ecosystems may also foster greater species adaptation to stressors. Freshwaters generally experience much greater thermal variation than marine systems, so freshwater ectotherms might be better adapted to temperature changes than those from more thermally-buffered marine ecosystems. For example, water fleas (Daphnia spp.) that are often focal species in lakes and ponds have been shown to be highly responsive (Colbourne et al., 2011) and capable of rapidly evolving in the face of environmental change (De Meester et al., 2011). Aquatic organisms also tend to be most sensitive to multiple stressor effects near their thermal tolerance limits (Heugens et al., 
2001), so more detrimental stressor interactions might be expected in marine ecosystems where species' ranges are often strongly aligned with their thermal limits (Pratchett et al., 2011; Sunday et al., 2012). Indeed, differences in how marine and freshwater ecosystems respond to similar stressors may depend on characteristics of the biological receptors and the environmental context, including the different communities, mechanisms, ecological networks and abiotic conditions present (Bancroft et al., 2007; Tylianakis et al., 2008; Segner et al., 2014).

Three stressor pair combinations had sufficient samples sizes $(n \geq 8$ for receptor categories within stressor pairs) for detailed analysis of interaction effects by level of biological organisation or organismal type. The mean interaction effect size remained significantly additive for nutrification paired with warming or habitat alteration (Fig. 4a) regardless of level of biological organisation or organism group. Contamination and warming had a significant antagonistic interaction overall (Fig. 4a) and at the organismal level $(d=-$ $0.77 ; 95 \% \mathrm{CI}=-1.3$ to $-0.3 ; n=10)$; however, the interaction was additive at the population $(d=-1.27 ; 95 \% \mathrm{CI}=-3.6$ to $0.4 ; n=11)$ and community $(d=-0.26 ; 95 \% \mathrm{CI}=-0.7$ to 0.2 ; $n=12)$ levels. Similarly, the mean interaction between contamination and warming became additive when considering only studies which measured impacts on vertebrates $(d=-0.26$; $95 \% \mathrm{CI}=-1.0$ to $0.5 ; n=12$ ). These results suggest that the type of organism and level of biological organisation are both important in determining and predicting the combined effects of specific stressor pairs.

\section{Reversal interactions as extreme ecological surprises}

Reversals (similar to 'mitigating synergisms' discussed by Piggott et al., 2015) were found in 34 out of 230 observations (14.78\%) included in our stressor pair analysis (i.e., the most inclusive response metric dataset). Although they were the least common type of 
interaction detected in our dataset of most inclusive end-points, reversal interactions warrant special consideration because they represent net effects that may differ markedly from those predicted by the typically assumed model of additivity (Piggott et al., 2015). Reversal interactions often involve the weaker of two stressors inverting the effect of the strongest. For instance, application of excess nutrients surprisingly reversed the toxic effect of atrazine on tadpoles as the additional resources likely permitted greater detoxification rates and stimulated growth, resulting in increased survival (Boone \& Bridges-Britton, 2006).

Our findings showed that the stressor most commonly associated with reversal interactions was warming (19.05\% of warming interactions; Fig. 4b). The greater likelihood of reversal interactions when a stressor is paired with higher temperatures might be related to the stimulatory effect of warming. As nearly all biological activity increases with warming (Brown et al., 2004), temperature changes arguably have the greatest potential to mediate the effects of other more damaging stressors. For example, Thompson et al., (2008) found that warming reversed the negative effect of excess nitrogen supply on growth by alpine phytoplankton, possibly because higher temperatures stimulated enzymatic conversion of nitrate and ammonia. In contrast, Linton et al., (1997) showed that higher temperatures could reverse the stimulatory effects of sub-lethal ammonia enrichment on juvenile rainbow trout (Oncorhynchus mykiss) by increasing metabolic costs to where ammonia detoxification and growth rates were reduced. In these cases, warming directly altered the mechanisms by which the dominant stressors affected the biological receptors. However, like other non-additive scenarios, reversals may also manifest from complex indirect interactions (e.g., Messner et al., 2013). Given the complexity of ecological responses to temperature changes (Petchey et al., 1999; O'Connor et al., 2009; Dossena et al., 2012; Stendera et al., 2012) and their potential role in generating non-additive interactions with other stressors (Crain et al., 2008; Harvey et al., 2013), we might then expect even more 'ecological surprises' in a warmer 
future.

\section{Conclusions}

We discovered a prevalence of antagonistic interactions between freshwater stressors across most receptor categories considered in our analysis (Table S2). Thus, there may exist a high potential for co-adaptation within freshwater ecosystems to minimize the net effects of multiple stressors. Alternatively, antagonism may be attributable to a high degree of asymmetry in the magnitude of independent effects between freshwater stressors (Folt et al., 1999). In this case, ranking the worst stressor driving an antagonistic interaction would be essential to forecasting their cumulative impacts on a freshwater ecosystem (Sala et al., 2000; Piggott et al., 2015). However, our evidence of predominantly antagonistic responses by freshwater organisms should not lessen the need to reduce exposure to stressors since their net effects were still mostly negative. The urgency of these findings are underscored by a recent global assessment that compared multiple stressor-induced average population declines of $76 \%$ among freshwater species to $39 \%$ among terrestrial and marine species since 1970 (WWF, 2014).

Non-additive interactions characterized $83 \%(192 / 230)$ of the cumulative impacts of multiple stressors in our most inclusive response metric dataset $(81 \%$ or $233 / 286$ in our full dataset). Mean interaction effect sizes varied significantly among stressor pairs and levels of receptor categories. Our analyses revealed different interactions for some stressor pairs (switching from antagonistic to additive, or vice versa) when only considering subsets of the data. This suggests that both stressor identity and characteristics of the ecological response (e.g., level of biological organisation and organism type) are essential in predicting interactions between multiple stressors in freshwater ecosystems.

Our findings have implications for conservation management of freshwater 
ecosystems. For stressor pairs that generate additive or synergistic effects, management focusing on a single stressor should render a positive outcome (Brown et al., 2013). However, in communities affected antagonistically by stressor pairs, both stressors may need to be removed or moderated to produce any substantial ecological recovery due to positive co-tolerance (Brown et al., 2013; Piggott et al., 2015).

Our findings evoke several testable hypotheses for further investigation. Firstly, the observed trend of stressor synergies increasing the productivity of primary producers suggests that higher temperatures, UVR exposure, and nutrient enrichment may jointly stimulate harmful algal blooms. Secondly, functional performance metrics appeared less sensitive overall than diversity metrics to dual stressors, highlighting the need for further investigation into the extent to which functional compensation occurs in stressed ecosystems. Thirdly, although we have demonstrated a clear predominance of antagonistic stressor interactions in freshwaters, further studies are needed to determine the specific underlying ecological mechanisms (e.g., asymmetry of stressor magnitudes, hard-selection for coadapted organisms, or similarity in behavioural or physiological acclimation). Finally, perhaps most interesting is our finding that multiple-stressor interactions differ between freshwaters and marine ecosystems and, although we have suggested several potential explanations, more research is needed to elucidate the specific physiological, genetic or environmental drivers behind these differences.

\section{Acknowledgements}

We are grateful to all the authors that responded with their data and our anonymous reviewers for their astute recommendations. We would also like to thank the Centre of Excellence for Invasion Biology (CIB) and the Canadian Aquatic Invasive Species Network (CAISN) II for their support towards this collaboration. 


\section{References}

Ban SS, Graham NA, Connolly SR (2014) Evidence for multiple stressor interactions and effects on coral reefs. Global Change Biology, 20, 681-697.

Bancroft BA, Baker NJ, Blaustein AR (2007) Effects of UVB radiation on marine and freshwater organisms: a synthesis through meta-analysis. Ecology Letters, 10, 332-345.

Boone MD, Bridges-Britton CM (2006) Examining multiple sublethal contaminants on the gray treefrog (Hyla versicolor): Effects of an insecticide, herbicide, and fertilizer. Environmental Toxicology and Chemistry, 25, 3261-3265.

Brown CJ, Saunders MI, Possingham HP, Richardson AJ (2013) Managing for interactions between local and global stressors of ecosystems. PLoS ONE, 8, e65765.

Brown JH, Gillooly JG, Allen AP, Savage VM, West GB (2004) Towards a metabolic theory of ecology. Ecology, 85, 1771-1789.

Christensen MR, Graham MD, Vinebrooke RD, Findlay DL, Paterson MJ, Turner MA (2006) Multiple anthropogenic stressors cause ecological surprises in boreal lakes. Global Change Biology, 12, 2316-2322.

Colbourne JK, Pfrender ME, Gilbert D et al. (2011) The ecoresponsive genome of Daphnia pulex. Science, 331, 555-561.

Crain CM, Kroeker K, Halpern BS (2008) Interactive and cumulative effects of multiple human stressors in marine systems. Ecology Letters, 11, 1304-1315.

Darling ES, Cote IM (2008) Quantifying the evidence for ecological synergies. Ecology Letters, 11, 1278-1286.

Dehedin A, Maazouzi C, Puijalon S, Marmonier P, Piscart C (2013) The combined effects of water level reduction and an increase in ammonia concentration on organic matter processing by key freshwater shredders in alluvial wetlands. Global Change Biology, 19, 763-774.

De Meester L, Van Doorslaer W, Geerts A, Orsini L, Stocks R (2011) Thermal genetic 
adaptation in the water flea Daphnia and its impact: An evolving metacommunity approach. Integrative and Comparative Biology, 51, 703-718.

Dossena M, Yvon-Durocher G, Grey J, Montoya JM, Perkins DM, Trimmer M, Woodward G (2012) Warming alters community size structure and ecosystem functioning. Proceedings of the Royal Society B-Biological Sciences, 279, 3011-3019.

Downing AL, Brown BL, Perrin EM, Keitt TH, Leibold MA (2008) Environmental fluctuations induce scale-dependent compensation and increase stability in plankton ecosystems. Ecology, 89, 3204-3214.

Doyle SA, Saros JE, Williamson CE (2005) Interactive effects of temperature and nutrient limitation on the response of alpine phytoplankton growth to ultraviolet radiation. Limnology and Oceanography, 50, 1362-1367.

Dudgeon D, Arthington AH, Gessner MO et al. (2006) Freshwater biodiversity: importance, threats, status and conservation challenges. Biological Reviews, 81, 163-182.

Elmqvist T, Folke, C, Nystrom M, Peterson G, Bengtsson J, Walker B, Norberg J (2003) Response diversity, ecosystem change, and resilience. Frontiers in Ecology and the Environment, 1, 488-494.

Fischer JM, Frost TM, Ives AR (2001) Compensatory dynamics in zooplankton community responses to acidification: measurement and mechanisms. Ecological Applications, 11, 10601072.

Folt CL, Chen CY, Moore MV, Burnaford J (1999) Synergism and antagonism among multiple stressors. Limnology and Oceanography, 44, 864-877.

Gonzalez A, Loreau M (2009) The causes and consequences of compensatory dynamics in ecological communities. Annual Review of Ecology, Evolution, and Systematics, 40, 393 414.

Gurevitch J, Hedges LV (2001) Meta-analysis: combining the results of independent 
experiments. In: Design and Analysis of Ecological Experiments, Second Edition (eds Scheiner SM, Gurevitch J), pp. 347-369. Oxford University Press, New York, USA. Gurevitch J, Morrison JA, Hedges LV (2000) The interaction between competition and predation: A meta-analysis of field experiments. The American Naturalist, 155, 435-453. Hall LW, Anderson RD (1995) The influence of salinity on the toxicity of various classes of chemicals to aquatic biota. Critical Reviews in Toxicology, 25, 281-346.

Harvey BP, Gwynn-Jones D, Moore PJ (2013) Meta-analysis reveals complex marine biological responses to the interactive effects of ocean acidification and warming. Ecology and Evolution, 4, 1016-1030.

Hering D, Carvalho L, Argillier C (2015) Managing aquatic ecosystems and water resources under multiple stress-An introduction to the MARS project. Science of the Total Environment, 503, 10-21.

Hillebrand H, Gurevitch J (2014) Meta-analysis results are unlikely to be biased by differences in variance and replication between ecological lab and field studies. Oikos, 123, 794-799.

Heugens EHW, Hendriks AJ, Dekker T, van Straalen NM, Admiraal W (2001) A review of the effects of multiple stressors on aquatic organisms and analysis of uncertainty factors for use in risk assessment. Critical Reviews in Toxicology, 31, 247-284.

Houlahan JE, Currie DJ, Cottenie K, et al. (2007) Compensatory dynamics are rate in natural ecological communities. Proceedings of the National Academy of Sciences of the United States of America, 104, 3273-3277.

Jenkins M (2003) Prospects for biodiversity. Science, 302, 1175-1177.

Klug JL, Fischer JM, Ives AR, Dennis B (2000) Compensatory dynamics in planktonic community responses to $\mathrm{pH}$ perturbations. Ecology, 81, 387-398.

Kosten S, Huszar VLM, Bécares E et al. (2012) Warmer climates boost cyanobacterial 
dominance in shallow lakes. Global Change Biology, 18, 118-126.

Lake PS (1990) Disturbing hard and soft bottom communities: a comparison of marine and freshwater environments. Australian Journal of Ecology, 15, 477-488.

Lindenmayer DB, Likens GE, Krebs CJ, Hobbs RJ (2010) Improved probability of detection of ecological "surprises". Proceedings of the National Academy of Sciences of the United States of America. 107, 21957-21962.

Linton TK, Reid SD, Wood CM (1997) The metabolic costs and physiological consequences to juvenile rainbow trout of a simulated summer warming scenario in the presence and absence of sublethal ammonia. Transactions of the American Fisheries Society, 126, 259272.

Mantyka-Pringle CS, Martin TG, Moffatt DB, Linke S, Rhodes JR (2014) Understanding and predicting the combined effects of climate change and land-use change on freshwater macroinvertebrates and fish. Journal of Applied Ecology, 51, 572-581.

Messner JS, MacLennan MM, Vinebrooke RD (2013) Higher temperatures enhance the effects of invasive sportfish on mountain zooplankton communities. Freshwater Biology, 58, $354-364$.

Mori AS, Furukawa T, Sasaki T (2012) Response diversity determines the resilience of ecosystems to environmental change. Biological Reviews, 88, 349-364.

Nyström M (2006) Redundancy and response diversity of functional groups: implications for the resilience of coral reefs. Ambio: A Journal of the Human Environment, 35, 30-35. O’Connor MI, Piehler MF, Leech DM, Anton A, Bruno JF (2009) Warming and resource availability shift food web structure and metabolism. PLoS Biology, 7, e1000178.

Ormerod SJ, Dobson M, Hildrew AG, Townsend CR (2010) Multiple stressors in freshwater ecosystems. Freshwater Biology, 55, 1-4.

Paine RT, Tegner MJ, Johnson EA (1998) Compounded perturbations yield ecological 
surprises. Ecosystems, 1, 535-545.

Parmesan C, Burrows MT, Duarte CM et al. (2013) Beyond climate change attribution in conservation and ecological research. Ecology Letters, 16, 58-71.

Petchey OL, McPhearson PT, Casey TM, Morin PJ (1999) Environmental warming alters food-web structure and ecosystem function. Nature, 402, 69-72.

Piggott, J.J., Townsend, C.R., Matthaei, C.D. (2015) Reconceptualizing synergism and antagonism among multiple stressors. Ecology and Evolution, 5, 1538-1547.

Pratchett MS, Bay LK, Gehrke PC (2011) Contribution of climate change to degradation and loss of critical fish habitats in Australian marine and freshwater environments. Marine and Freshwater Research, 62, 1062-1081.

Przeslawski R, Byrne M, Mellin C (2015) A review and meta-analysis of the effects of multiple abiotic stressors on marine embryos and larvae. Global Change Biology, online first. R Core Team (2014) R: A language and environment for statistical computing. R Foundation for Statistical Computing: Version 3.0.3, Vienna, Austria. URL: http://www.R-project.org/. Root TL, Price JT, Hall KR, Schneider SH, Rosenzweig C, Pounds JA (2003) Fingerprints of global warming on wild animals and plants. Nature, 421, 57-60.

Rosenberg MS, Adams DC, Gurevitch J (2000) MetaWin: Statistical software for metaanalysis: Version 2.1.

Sala OE, Chapin III FS, Armesto JJ et al. (2000) Global biodiversity scenarios for the year 2100. Science, 287, 1770-1774.

Segner H, Schmitt-Jansen M, Sabater S (2014) Assessing the impact of multiple stressors on aquatic biota: the receptor's side matters. Environmental Science and Technology, 48, 76907696.

Smith MD, Knapp AK (2003) Dominant species maintain ecosystem function with nonrandom species loss. Ecology Letters, 6, 509-517. 
Strain EMA, Thomson RJ, Micheli F, Mancuso FP and Airoldi L (2014) Identifying the interacting roles of stressors in driving the global loss of canopy-forming to mat-forming algae in marine ecosystems. Global Change Biology, 20, 3300-3312.

Staudt A, Leidner AK, Howard J et al. (2013) The added complications of climate change: understanding and managing biodiversity and ecosystems. Frontiers in Ecology and the Environment, 11, 494-501.

Steinger T, Roy BA, Stanton ML (2003) Evolution in stressful environments II. Adaptive value and costs of plasticity in response to low light in Sinapis arvensis. Journal of Evolutionary Biology, 16, 313-323.

Stendera S, Adrian R, Bonada N et al. (2012) Drivers and stressors of freshwater biodiversity patterns across different ecosystems and scales: a review. Hydrobiologia, 696, 1-28.

Sunday JM, Bates AE, Dulvy NK (2012) Thermal tolerance and the global redistribution of animals. Nature Climate Change, 2, 686-690.

Thompson PL, St-Jacques MC, Vinebrooke RD (2008) Impacts of climate warming and nitrogen deposition on alpine plankton in lake and pond habitats: an in vitro experiment. Arctic Antarctic and Alpine Research, 40, 192-198.

Tylianakis JM, Didham RK, Bascompte J, Wardle DA (2008) Global change and species interactions in terrestrial ecosystems. Ecology Letters, 11, 1351-1363.

Vaz-Pinto F, Olabarria C, Gestoso I, Cacabelos E, Incera M, Arenas F (2013) Functional diversity and climate change: effects on the invisibility of macroalgal assemblages.

Biological Invasions, 15, 1833-1846.

Vucic-Pestic O, Ehnes RB, Rall BC, Brose U (2011) Warming up the system: higher predator feeding rates but lower energetic efficiencies. Global Change Biology, 17, 1301-1310.

Vinebrooke RD, Schindler DW, Findlay DL, Turner MA, Paterson M, Mills KH (2003) Trophic dependence of ecosystem resistance and species compensation in experimentally 
acidified lake 302S (Canada). Ecosystems, 6, 101-113.

Vinebrooke RD, Cottingham KL, Norberg J, Scheffer M, Dodson SI, Maberly SC, Sommer U (2004) Impacts of multiple stressors on biodiversity and ecosystem functioning: the role of species co-tolerance. Oikos, 104, 451-457.

Williamson CE, Saros JE, Schindler DW (2009) Sentinels of Change. Science, 323, 887-888. WWF (2014) Living Plant Report 2014: species and spaces, people and places (eds McLellan R, Iyengar L, Jeffries B, Oerlemans N). World Wide Fund for Nature, Gland, Switzerland.

\section{Supporting Information Legends}

1. Detailed methods: Details of data search and selection criteria, effect size calculation and interpretation, weighted meta-analyses and vote-counting methodology.

2. Meta-analysis tables and references: Interaction effect sizes and related information for each study used in our meta-analyses (Table S1), mean interaction effect sizes and details of meta-analytic models used in our analyses (Table S2) and a detailed list of data references.

3. Robustness of meta-analytic results: Detailed analysis of potential publication bias and sensitivity of our results to variations in sample sizes and effect size outliers. 